CIC. Cuadernos de Información y Comunicación ISSN: 1135-7991

http://dx.doi.org/10.5209/ciyc.64639

\title{
Plataformas en línea y diversidad audiovisual: desafíos para el mercado español ${ }^{1}$
}

\author{
$\mathrm{M}^{\mathrm{a}}$ Trinidad García Leiva
}

Recibido: 10 de abril de 2019/ Aceptado: 25 de mayo de 2019

Resumen. Partiendo de problematizar el concepto de plataforma, en relación con el de industria audiovisual, este artículo ofrece un mapa de aquellas que comercializan en España contenidos audiovisuales OTT, bajo demanda y mediados por abono. El objetivo es elaborar una clasificación y explicar cuál es su perfil empresarial para evidenciar que las 'plataformas españolas' manifiestan una clara atomización empresarial mientras que el mercado es dominado por agentes establecidos en el exterior. Los mismos están vinculados a los llamados GAFAM y a corporaciones mundiales de medios y entretenimiento, además de incluir a Netflix, claro está. Se concluye sobre las oportunidades y las amenazas que estas plataformas presentan para la diversidad de/en la industria audiovisual española.

Palabras clave: Industria audiovisual, televisión, cine, Netflix, concentración, OTT.

\section{[en] Online Platforms and Audiovisual Diversity: Challenges for the Spanish market}

\begin{abstract}
Taking the concept of platform as a point of departure, in relation to that of audiovisual industry, this article presents a map of those that offer in Spain audiovisual productions OTT, by payment and on demand. The aim is to classify and explain its business profile, in order to show that 'Spanish platforms' are atomized whereas the market is controlled by few platforms based abroad. These are linked to the so-called GAFAM or to media and entertainment corporations; apart from including Netflix. It concludes with a reflection on the opportunities and challenges that OTT platforms present for the diversity of/in the Spanish audiovisual industry.
\end{abstract}

Keywords: Audiovisual Industry, Television, Film, Netflix, Concentration, OTT.

Sumario. 1. Introducción. 2. Las plataformas audiovisuales. 3. España: mucho (y muchas) más que Netflix. 3.1. ¿De la ebullición a la concentración?. 3.2. ¿Atomización empresarial vs. hegemonía global?. 3.3. ¿Hay un 'efecto Netflix’?. 4. A modo de conclusión. 5. Referencias bibliográficas.

Cómo citar: García Leiva, $\mathrm{M}^{\mathrm{a}} \mathrm{T}$. (2019). Plataformas en línea y diversidad audiovisual: desafíos para el mercado español, en CIC. Cuadernos de Información y Comunicación 24, 73-93.

1 Universidad Carlos III de Madrid. Este artículo se enmarca en el proyecto de investigación 'Diversidad audiovisual y plataformas en línea: el caso Netflix' (CSO2017-83539-R), financiado por la Agencia Estatal de Investigación (AEI), en el marco del Programa Estatal de I+D+i Orientada a los Retos de la Sociedad del Ministerio de Ciencia, Innovación y Universidades de España, y por el Fondo Europeo de Desarrollo Regional (FEDER) de la Unión Europea. 


\section{Introducción}

Las plataformas que comercializan contenidos audiovisuales a través de la Internet pública abierta (más conocidas por su denominación en inglés over the top, y las siglas OTT), producidos y/o adquiridos para tal fin, están alterando la configuración de las industrias audiovisuales, digitales y analógicas, redefiniendo el rol de los agentes existentes al disputar espectadores, suscriptores y anunciantes.

Según BCG Analysis (2016), a mediados de esta década el segmento del audiovisual OTT había crecido hasta los 25.000 millones de dólares estadounidenses de ganancias anuales, generadas fundamentalmente por un puñado de agentes globales con origen en Estados Unidos. Si bien esta consultora computaba más de 500 proveedores de servicios audiovisuales OTT en todo el mundo, sólo cinco empresas de este tipo operaban a escala internacional - Netflix, Amazon, Hulu, HBO y YouTube - concentrando cerca de la mitad del total de las ganancias (además de estar especialmente bien posicionadas para captar el crecimiento futuro por tener ventajas competitivas en áreas tales como la innovación tecnológica o la escala en la creación de contenidos).

Más específicamente, las plataformas que ofrecen audiovisual OTT, bajo demanda y mediados por abono (suscripción o pago por visión), son señaladas por distintos estudios como especialmente disruptivas y como las que lideran el crecimiento del mercado audiovisual en Internet (Grece et al., 2015; IDATE, 2016; BCG Analysis, 2016; PwC, 2018a). Según Digital TV Research (2018), las suscripciones mundiales a este tipo de plataformas aumentarán en 409 millones entre 2017 y 2023, ascendiendo en dicho año a un total de 777 millones.

Este crecimiento también se registra en España desde el primer semestre de 2017, cuando el Panel de Hogares de la Comisión Nacional del Mercado de la Competencia (CNMC, 2017) detectó un rápido aumento de las plataformas de pago para ver contenidos audiovisuales en línea: casi 1 de cada 4 hogares con acceso a Internet era usuario de alguno de estos servicios de vídeo en streaming. El crecimiento más llamativo era el de Netflix, que en tan sólo seis meses (diciembre 2016-junio 2017) había duplicado su número de usuarios: a junio de 2017 tenía 1.163 .000 suscriptores (7,3\% del total de hogares con acceso a Internet). Según esta misma fuente, un año después, Netflix acumulaba 2 millones de abonados, acortando distancias con Movistar+ (CNMC, 2018b).

En este contexto de aumento de la cantidad de contenidos disponibles debido a la irrupción de la oferta de nuevos agentes, en el que se consolidan además otras fuerzas que configuran la forma de consumir contenidos audiovisuales (el consumo a medida, propiciado por la expansión de la banda ancha y el acceso a contenidos en línea; y el consumo multi-pantalla, descentralizado y ubicuo, fomentado por la existencia de diversos dispositivos; Fundación Telefónica, 2017), parece importante estudiar el papel que juegan las plataformas en línea para la diversidad de las industrias audiovisuales.

Partiendo de problematizar el concepto de plataforma en relación con el de industria audiovisual, este artículo ofrece un mapa de las que comercializan en España contenidos audiovisuales OTT, bajo demanda y mediados por abono, con la intención de elaborar una tipología y explicar cuál es su perfil empresarial. Se trata de destacar, en última instancia, tanto las oportunidades como las amenazas que presentan para la diversidad de/en la industria audiovisual española. 
Al fin y al cabo, aunque se trate de actores que constituyen vectores de desarrollo comercial y catalizadores de innovación, las plataformas también fomentan y median en diversas formas de interacción social, por lo que su talla y prácticas no deben ahogar a los competidores locales y/o más pequeños ni seguir beneficiándose del vacío legal que las sitúa fuera de la órbita de los órganos reguladores nacionales.

Cabe aclarar que conceptualmente pensar la diversidad de y en las industrias audiovisuales depende de una multiplicidad de factores. A fin de evaluarla deben tenerse en consideración, como mínimo, que (Albornoz y García Leiva, 2017a):

a. La capacidad de producción, distribución y exhibición/emisión de contenidos audiovisuales no esté concentrada en un número reducido de agentes y que estos agentes sean de diferentes tipos de titularidad, tamaño y origen geográfico.

b. Los contenidos audiovisuales exhiban diferencias de variedad, balance y disparidad (Stirling, 1998 y 2007) en términos de valores, identidad y estética. Estos deben reflejar a los múltiples grupos que conviven en una determinada sociedad y hacerse eco de culturas foráneas.

c. Los ciudadanos puedan acceder y elegir entre un elevado número de contenidos audiovisuales e, incluso, puedan crearlos y difundirlos.

\section{Las plataformas audiovisuales}

Más que cambiar radicalmente la configuración económica de la cultura, la digitalización ha contribuido a cuestionar los equilibrios de poder existentes y las relaciones (inter)sectoriales, al permitir modelos alternativos de creación, producción, promoción, distribución y consumo (De Voldere, 2017; Frau-Meigs y Kiyindou, 2014; A1bornoz y García Leiva, 2017b y 2019). Las denominadas plataformas en línea han surgido en este complejo escenario y son parte de él.

Sin embargo, no existe consenso acerca de su definición (ver por ejemplo: Cabrera y Valais, 2016). De hecho, la amplitud de los usos del término plataforma en relación a determinados operadores y/o servicios de Internet ha sido analizada críticamente como un proceso discursivo, política y empresarialmente intencionado, que permite a actores poderosos - como Google- perseguir sus objetivos ejerciendo de intermediarios sin entrar en conflicto con los múltiples agentes con los que intervienen: gobiernos, empresas, usuarios, anunciantes, etc. (Gillespie, 2010). Desde un punto de vista económico, son mercados de 'dos caras' o 'múltiples caras' en los que dos o más tipos de usuarios (compradores y vendedores, anunciantes, desarrolladores de software o usuarios de redes sociales) se reúnen a propósito de la plataforma para dar lugar a una transacción o intercambio (Martens, 2016).

Lo cierto es que el término plataforma en línea suele referir a ejemplos que presentan distintas formas y tamaños, y que cubren una amplia gama de actividades: publicidad en línea, motores de búsqueda, redes sociales y medios de difusión de contenidos culturales, servicios de comunicación, aplicaciones, sistemas de pago y economía colaborativa (CE, 2016). Algunas plataformas reconocidas son: eBay, Amazon Marketplace, Google, Bing Search, Facebook, YouTube, Google Play, App Store, BlaBla Car y Über. 
Según la Comisión Europea, pese a sus diferencias todas comparten ciertas características (CE, 2016: 2):

a. tienen capacidad para crear y modelar nuevos mercados, planteando a los agentes existentes un verdadero desafío, así como para organizar nuevas formas de participación o para efectuar en línea negocios consistentes en la recogida, tratamiento y edición de grandes cantidades de datos;

b. actúan en mercados plurifacéticos pero con diversos grados de control sobre las interacciones directas entre grupos de usuarios;

c. se benefician de los 'efectos de red', lo que significa en sentido amplio que el valor del servicio aumenta con el número de sus usuarios;

d. suelen apoyarse en las TICs para llegar a sus usuarios de forma instantánea y sin esfuerzo;

e. y desempeñan un papel de crucial importancia en la creación de valor digital, facilitando nuevos proyectos empresariales y creando nuevas dependencias estratégicas.

En el ámbito audiovisual existen diversos tipos de plataformas comercializadoras de contenidos, entre las cuales una primera distinción permite diferenciar a las que producen y/o adquieren los contenidos ofertados -Netflix, Spotify...- de las que simplemente los agregan -YouTube, TuneIn...-(CISAC, 2017). Asimismo es posible clasificar estas plataformas en función de si los contenidos se ofertan a través de la Internet pública abierta (OTT) o no.

Si bien, como ocurre con la expresión plataformas digitales, no existe acuerdo unánime sobre la definición y alcance del concepto OTT, según la CNMC (2015) estos servicios pueden definirse como aquellos que se ofertan sobre Internet sin mediar control alguno ni gestión específica por parte de los operadores de red. Así, Netflix o YouTube son ejemplos de agentes que prestan servicios audiovisuales OTT, mientras que los conocidos como servicios de televisión IP (la oferta de televisión en línea de un operador de cable o de satélite, como Movistar en España) se distribuyen a sus clientes finales a través de una infraestructura gestionada por un operador.

Pero cuidado porque, como señala Prado (2017), las ofertas OTT no excluyen la posibilidad de limitar el acceso a determinados contenidos, ya sea con la finalidad de proteger públicos sensibles o derechos de propiedad intelectual (geoblocking), o en función de hacer posible un modelo de negocio particular (pay OTT). No hay que olvidar que si bien el OTT se popularizó primero como territorio privilegiado para contenidos generados por los usuarios (caso YouTube), con el paso del tiempo ha tenido lugar un desplazamiento hacia agentes industriales que articulan contenidos profesionales poniendo en marcha un sistema de financiación basado esencialmente en la publicidad, la suscripción y/o la compra y/o el alquiler de contenidos (Prado, 2017).

Este trabajo se centra en un grupo muy específico de los servicios que la recientemente modificada Directiva de servicios de comunicación audiovisual define como bajo demanda (es decir, no lineales o a petición ${ }^{2}$ ), los cuales se explican y ejemplifi-

Directiva 2018/1808 del Parlamento Europeo y del Consejo de 14 de noviembre de 2018. Diario Oficial de la Unión Europea de 28 de noviembre de 2018. L303/69. 
can en la tabla 1, junto con otros criterios utilizados para su clasificación en el siguiente apartado. Tales criterios refieren a los modelos de negocio que financian el servicio, el tipo de contenidos puestos a disposición, y el país en relación al cual el operador del servicio lo gestiona y/u ofrece.

Tabla 1: Clasificación de servicios audiovisuales bajo demanda.

\begin{tabular}{|c|c|}
\hline \multicolumn{2}{|r|}{ Según nueva Directiva de servicios de comunicación audiovisual } \\
\hline \multirow[t]{3}{*}{ Incluidos } & $\begin{array}{l}\text { Catch-up } T V \text { : puesta a disposición de contenidos ya emitidos de forma } \\
\text { lineal, en general a cargo de operadores tradicionales de televisión. } \\
\text { Ejemplo: Mitele. }\end{array}$ \\
\hline & $\begin{array}{l}\text { Services on sharing platforms: contenidos de/en plataformas para compartir } \\
\text { vídeo (cuya funcionalidad esencial sea proveer programas y vídeos generados } \\
\text { por usuarios). Se incluyen los que se ponen a disposición de manera separada y } \\
\text { restringida. Ejemplos: YouTube y YouTube Movies. }\end{array}$ \\
\hline & $\begin{array}{l}\text { Video on demand services: puesta a disposición de un catálogo de } \\
\text { producciones cinematográficas y/o televisivas, independientemente de } \\
\text { difusiones anteriores. Ejemplo: Netflix. }\end{array}$ \\
\hline \multirow[t]{2}{*}{ No incluidos } & $\begin{array}{l}\text { Sitios web promocionales que incluyen contenido audiovisual. Ejemplo: Coke } \\
\text { TV. }\end{array}$ \\
\hline & $\begin{array}{l}\text { Sitios web de información que incluyen contenido audiovisual. Ejemplo: } \\
\text { elpais.com }\end{array}$ \\
\hline \multicolumn{2}{|r|}{ Según modelos de negocio } \\
\hline TVoD & $\begin{array}{l}\text { Transactional video on demand en inglés, también conocido como pago por } \\
\text { visión (pay per view), refiere a la transacción que ha de tener lugar para que } \\
\text { el usuario acceda a un contenido específico, durante un tiempo determinado, } \\
\text { a cambio de una tarifa predefinida. Es decir, puede referir al alquiler o a la } \\
\text { compra en línea. }\end{array}$ \\
\hline PVoD & $\begin{array}{l}\text { Pack video on demand en inglés, refiere al acceso, a través una misma y única } \\
\text { transacción, a un número limitado de producciones, en general por un periodo } \\
\text { determinado. }\end{array}$ \\
\hline SVoD & $\begin{array}{l}\text { Subscription video on demand en inglés, refiere al acceso ilimitado a una } \\
\text { librería o catálogo de producciones a cambio de una tarifa mensual. }\end{array}$ \\
\hline $\mathrm{AVoD}$ & $\begin{array}{l}\text { Advertising-supported video on demand, también conocido en inglés como } \\
\text { free video on demand, ofrece el acceso a los contenidos de forma 'gratuita' (los } \\
\text { mismos se financian a partir de la inclusión de publicidad). }\end{array}$ \\
\hline Freemium & $\begin{array}{l}\text { Conjunción de las palabras free y premium, refiere a la combinación de una } \\
\text { oferta compuesta, por un lado, por contenidos básicos / generalistas, de } \\
\text { acceso gratuito y, por otro, por contenidos avanzados / especializados, de } \\
\text { pago. }\end{array}$ \\
\hline
\end{tabular}




\begin{tabular}{|c|c|}
\hline \multicolumn{2}{|r|}{ Según tipo de contenidos } \\
\hline Generalista & $\begin{array}{l}\text { Contenidos variados: cine, ficción televisiva, TV shows, documentales, } \\
\text { animación, infantil, etc. }\end{array}$ \\
\hline $\begin{array}{l}\text { Cine y/o ficción } \\
\text { TV }\end{array}$ & $\begin{array}{l}\text { Oferta de contenidos cinematográficos y/o televisivos de ficción, en sus } \\
\text { diferentes formatos y extensiones (largometrajes, cortos, TV movies, series, } \\
\text { miniseries...). }\end{array}$ \\
\hline Documentales & Contenidos especializados en programas documentales. \\
\hline $\begin{array}{l}\text { Infantil/ } \\
\text { animación }\end{array}$ & $\begin{array}{l}\text { Contenidos especializados en obras infantiles y/o de animación (especialmente } \\
\text { manga). }\end{array}$ \\
\hline Musical & $\begin{array}{l}\text { Oferta centrada en programas musicales (vídeos musicales o eventos musicales } \\
\text { grabados). }\end{array}$ \\
\hline Adulto & Contenidos dedicados a mayores de 18 años. \\
\hline $\begin{array}{l}\text { Estilo vida/ } \\
\text { salud }\end{array}$ & Oferta relacionada con la salud, el bienestar y los estilos de vida. \\
\hline Deportes & Contenidos relacionados con el deporte y eventos deportivos grabados. \\
\hline Otros & $\begin{array}{l}\text { Producciones especializadas en algún género como, por ejemplo, el cine de } \\
\text { terror o el cine clásico. }\end{array}$ \\
\hline \multicolumn{2}{|r|}{ Según país de establecimiento de y/o de acceso al servicio } \\
\hline $\begin{array}{l}\text { Establecidos en } \\
\text { el país }\end{array}$ & $\begin{array}{l}\text { Aquellos servicios cuyo proveedor se encuentra legalmente establecido en el } \\
\text { país en el que se ofrecen. }\end{array}$ \\
\hline $\begin{array}{l}\text { Ofertados en el } \\
\text { país }\end{array}$ & $\begin{array}{l}\text { Aquellos servicios cuyo proveedor se encuentra legalmente establecido en un } \\
\text { país diferente al que se ofrecen. }\end{array}$ \\
\hline
\end{tabular}

Fuente: elaboración propia a partir de criterios utilizados por el Observatorio Audiovisual Europeo.

\section{España: mucho (y muchas) más que Netflix}

Los datos de la CNMC (2018a y 2018b) ponen de manifiesto tanto el crecimiento del mercado de la televisión de pago en general, desde 2016, de la mano de Movistar+ y los paquetes de servicios integrados de Telefónica (ofertas combinadas de audiovisual y telecomunicaciones), como el salto importante de penetración de mercado que registran las plataformas de pago OTT. Los últimos (y únicos) datos disponibles así lo demuestran (ver tabla 2).

Y si bien es cierto que este crecimiento coincide con el desembarco en el mercado español de plataformas de origen foráneo (a la llegada de Netflix en noviembre de 2015 siguieron la de HBO y Amazon en diciembre de 2016, y la de Sky en septiembre de 2017), más allá de las valoraciones entusiastas y coyunturales de este fenómeno, así como de las previsiones optimistas sobre su evolución futura ( $\mathrm{PwC}, 2018 \mathrm{a}$ y 2018b), es necesario un análisis detallado que aquilate quiénes y cómo son las plataformas que comercializan en España contenidos audiovisuales OTT, bajo demanda y mediados por abono. Este apartado ofrece un mapa de las mismas para retratar y clasificar su perfil empresarial. 
Tabla 2. Uso de plataformas de pago para ver contenidos audiovisuales en línea (porcentaje de hogares, 2016-18).

\begin{tabular}{|l|r|r|r|r|r|}
\hline \multirow{2}{*}{ Servicio } & \multicolumn{2}{|c|}{$\mathbf{2 0 1 6}$} & \multicolumn{2}{c|}{$\mathbf{2 0 1 7}$} & \multicolumn{1}{c|}{$\mathbf{2 0 1 8}$} \\
\cline { 2 - 6 } & \multicolumn{1}{|c|}{ T2 } & \multicolumn{1}{c|}{ T4 } & \multicolumn{1}{c|}{ T2 } & \multicolumn{1}{c|}{ T4 } & \multicolumn{1}{c|}{ T2 } \\
\hline No uso & $89.3 \%$ & $88.0 \%$ & $77.5 \%$ & $70.2 \%$ & $66.3 \%$ \\
\hline Rakuten TV & $1.1 \%$ & $1.3 \%$ & $0.8 \%$ & $1.0 \%$ & $0.9 \%$ \\
\hline Netflix & $1.8 \%$ & $3.4 \%$ & $7.3 \%$ & $9.1 \%$ & $12.5 \%$ \\
\hline Movistar+ en dispositivos & $7.8 \%$ & $7.6 \%$ & $12.6 \%$ & $13.5 \%$ & $13.4 \%$ \\
\hline Vodafone TV online & NA & NA & NA & $5.9 \%$ & $5.8 \%$ \\
\hline App Orange TV & NA & NA & NA & $2.8 \%$ & $4.5 \%$ \\
\hline HBO & NA & NA & $2.6 \%$ & $2.3 \%$ & $2.9 \%$ \\
\hline Amazon Prime & NA & NA & $1.1 \%$ & $3.5 \%$ & $4.0 \%$ \\
\hline beIN CONNECT/Total Channel & $0.2 \%$ & $0.2 \%$ & $1.2 \%$ & $0.8 \%$ & $2.3 \%$ \\
\hline Filmin & $0.1 \%$ & $0.2 \%$ & $0.1 \%$ & NA & NA \\
\hline Sky & NA & NA & NA & NA & $0.7 \%$ \\
\hline Otras & $0.5 \%$ & $0.6 \%$ & $0.9 \%$ & $0.3 \%$ & $0.6 \%$ \\
\hline
\end{tabular}

Notas: NA: No aplica. T: trimestre.

Fuente: CNMC, Panel de Hogares (posible respuesta múltiple).

\section{1. ¿De la ebullición a la concentración?}

Siguiendo la estela de trabajos anteriores (García Leiva, 2017), así como la información proporcionada por la base de datos MAVISE, del Observatorio Audiovisual Europeo, el informe de Findanygame (2017) sobre plataformas digitales de cine y series en España, y el sitio web La Pantalla Digital, cualquier retrato de situación debe empezar por señalar que los consumidores españoles pueden acceder a 41 plataformas audiovisuales de pago bajo demanda, disponibles OTT (ver tabla 3 ).

En este grupo se detectan 17 plataformas con sede en España; mientras que entre las 24 restantes son mayoría las localizadas en un mismo país: Estados Unidos (10). Como se analizará más adelante, no hay un modelo de negocio dominante sino distintas modalidades (y combinaciones) de comercialización de contenidos, las cuales se pueden poner en relación tanto con la talla del proveedor del servicio como con el tipo de contenido ofrecido. Dichas modalidades, que a veces incluyen también opciones 'gratis', pueden desdoblarse a su vez en 'planes' definidos, generalmente, en función del número de usuarios/pantallas a las que se da acceso, así como de la cali$\mathrm{dad} /$ funcionalidades asociadas al visionado.

Evidentemente un mapa es siempre una foto fija, que por ello presenta tanto ventajas como desventajas para estudiar la diversidad del audiovisual en línea (García Leiva, 2016). Sin embargo, si el ejercicio se efectúa con periodicidad, es posible detectar tendencias e hitos. Precisamente, resultado de haber efectuado en el pasado un mapeo de las plataformas audiovisuales de pago bajo demanda, disponibles en España OTT, cabe hacer las siguientes observaciones que permitan, al mismo tiempo, una periodización. 
Las plataformas más antiguas surgieron en España y para el mercado español a mediados de los años 2000, cuando se fraguaron iniciativas como las de ADNStream, Televeo, Filmotech y Filmin, que vieron la luz a partir de 2007. De todas ellas sólo funciona de modo solvente la última, ya que las primeras tienen una talla modesta y Filmotech cesó su actividad el 1 de febrero de 2019. Portal creado por EGEDA (Entidad de Gestión de Derechos de los Productores Audiovisuales), el mismo llegó a tener en 2018 casi 2.200 títulos en su catálogo con una oferta por suscripción de $6,95 €$ mensuales y de pago por visión desde $0,50 €$.

Una segunda oleada de iniciativas locales se produjo con el cambio de década, cuando entre 2010 y 2013 surgieron las aventuras de los operadores de televisión tradicional (Mitele y Atresplayer, entonces A3Player y antes Nubeox), nuevas iniciativas comerciales (Cineclick, Benecé online, Waki - hoy Rakuten - Youzee, Tucut, Booquo y Mivideoclub.com), así como propuestas de nicho (IN-EDIT TV, 400 Films y Feelmakers). Como se explica a continuación muchas ya no existen.

El recorrido de 400 Films, que se planteó en competencia con Filmin, se limitó a los años 2012-15. A su vez, Booquo, puesta en marcha en 2012 por el Círculo de Lectores (entonces de los grupos Bertelsmann y Planeta a partes iguales), pasó de ser la primera plataforma en ofrecer películas y libros bajo demanda, bajo suscripción o compra, a ofrecer sólo libros desde septiembre de 2013, cuando se relanzó como Nubico (con la participación de Telefónica). Youzee, por su parte, estuvo en pie poco más de un año (201213), participada al 75\% por Yelmo Cines, y ofreciendo en su lanzamiento unos 500 títulos por una suscripción mensual de $7 €$ o un alquiler desde 1,5€. En 2013 se lanzó Tucut, con el reclamo de ser el videoclub online centrado en cine español, mediante suscripción, combinado con el componente social de la red. No superó los tres años de andadura. Mivideoclub.com acabó reconvirtiéndose en Videoping: solución para distribuidoras o productoras que facilita la comercialización en Internet de contenidos audiovisuales.

Cabe recordar que para esas fecha ya funcionaban todas las opciones de alquiler de estrenos comerciales y títulos de las majors a través de las tiendas virtuales de Microsoft, iTunes y Sony, además de estar disponibles las opciones de comercialización de Google y YouTube. Aunque de forma singular deben destacarse, durante esta fase, los lanzamientos de Vimeo on Demand, realeyz (ambos en 2009) y MUBI (2010). Definibles por su grado de especialización, abrieron la puerta a otras ofertas foráneas que desde ese momento empezaron a multiplicarse. Entre 2012 y 2014 aparecieron: WolfeOnDemand, EROSNOW, spuul, Crunchyroll, Full Moon, iROKOtv y FilmDooc, de nicho, y Voddler, dedicada a títulos comerciales, así como clásicos y algunas referencias europeas, con una aproximación generalista. Este último servicio, de origen sueco, lanzado en marzo de 2012, no estuvo operativo más de dos años.

En pocas palabras, la primera parte de la década de 2010 estuvo plagada de lanzamientos y cierres. Evidentemente esta periodización no menciona de modo exhaustivo a todas las iniciativas que han estado disponibles en el mercado español, ya que ello escapa a la extensión y foco de este trabajo. Sin embargo, para ofrecer una idea más o menos acabada de la efervescencia e inestabilidad de este segmento de la industria audiovisual, basta relacionar los servicios que se encontraban a disposición en 2014, según el relevamiento efectuado entonces por el sitio Findanygame ${ }^{3}$ : Anijapan, AfricaFilms.tv, buong.tv, Cine Accesible, CineOnline, Engeldroid, Videomatic, viveX, Erotic Movie House, o Bollyvod. Ninguno funciona hoy.

Ver https://www.hermenaute.com/articles_judex.php?id_article=2373 
Tabla 3. Plataformas audiovisuales de pago bajo demanda disponibles en España OTT - Mapa (abril de 2019).

\begin{tabular}{|c|c|c|c|c|}
\hline Servicio & Proveedor & Propietario & \multicolumn{2}{|c|}{$\begin{array}{l}\text { Lanzamiento } \\
\text { y país } \\
\text { establecimiento }\end{array}$} \\
\hline Rakuten TV (ES) & Rakuten TV Europe SLU & Rakuten Inc. & 2011 & España \\
\hline Mitele & Mediaset & Mediaset SPA & 2011 & España \\
\hline Atresplayer & Atresmedia & $\begin{array}{l}\text { Atresmedia Corporación } \\
\text { SA }\end{array}$ & 2013 & España \\
\hline Filmin & \multicolumn{2}{|l|}{ Comunidad Filmin S.L. } & 2008 & España \\
\hline FlixOlé & \multicolumn{2}{|l|}{ Lomatena Investments S.L. } & 2018 & España \\
\hline Cineclick & \multicolumn{2}{|l|}{ Cineclick SL } & 2010 & España \\
\hline ADNStream & ADNStream SL & $\begin{array}{l}\text { Fractal Internet } \\
\text { Consultancy }\end{array}$ & 2007 & España \\
\hline Televeo & Televeo Broadcasting SL & $\begin{array}{l}\text { M4V Interactive } \\
\text { Business }\end{array}$ & 2007 & España \\
\hline Benecé online & \multicolumn{2}{|c|}{ Benecé Produccións SL (productora audiovisual) } & 2012 & España \\
\hline Feelmakers & \multicolumn{2}{|l|}{ Content Line SL } & 2013 & España \\
\hline GuiDoc & GuiDoc & Víctor Correal (fundador) & 2016 & España \\
\hline $\begin{array}{l}\text { Tus Telenovelas } \\
\text { Online }\end{array}$ & Fractal Media & $\begin{array}{l}\text { Fractal Internet } \\
\text { Consultancy SL }\end{array}$ & $\mathrm{s} / \mathrm{d}$ & España \\
\hline Mujeres de Cine & \multicolumn{2}{|l|}{ Mujeres de Cine (colectivo) } & 2019 & España \\
\hline Cine Autor & \multicolumn{2}{|c|}{ Screen Art Films SL (productora cinematográfica) } & 2019 & España \\
\hline IN-EDIT TV & \multicolumn{2}{|c|}{ Inedit Producciones SL (productora festival IN-EDIT) } & 2011 & España \\
\hline márgenes & \multicolumn{2}{|c|}{$\begin{array}{l}\text { River Events Highway (productora y distribuidora } \\
\text { cine) }\end{array}$} & 2016 & España \\
\hline NYX Channel & NYX Channel SL & $\begin{array}{l}\text { Panteo Films SL } \\
\text { (doblaje) }\end{array}$ & 2017 & España \\
\hline $\begin{array}{l}\text { Microsoft Store - } \\
\text { Movies \& TV (ES) }\end{array}$ & Microsoft Inc. & Microsoft Inc. & 2008 & $\begin{array}{l}\text { Estados } \\
\text { Unidos }\end{array}$ \\
\hline $\begin{array}{l}\text { Google Play } \\
\text { Movies (ES) }\end{array}$ & Google Inc. & Alphabet Inc. & 2012 & $\begin{array}{l}\text { Estados } \\
\text { Unidos }\end{array}$ \\
\hline iTunes Store (ES) & iTunes Sarl. & Apple Inc. & 2010 & Irlanda \\
\hline Play Station Store & $\begin{array}{l}\text { Sony Entertainment } \\
\text { Network (ES) }\end{array}$ & Sony Corp. & 2009 & $\begin{array}{l}\text { Reino } \\
\text { Unido }\end{array}$ \\
\hline Prime Video (ES) & Amazon Instant Vídeo Ltd. & Amazon Inc. & 2018 & $\begin{array}{l}\text { Reino } \\
\text { Unido }\end{array}$ \\
\hline YouTube Movies & YouTube LLC & Alphabet Inc. & 2011 & EE.UU. \\
\hline HBO España & HBO Nordic AB & Time Warner Inc. & 2016 & Suecia \\
\hline Netflix España & Netflix International BV & Netflix Inc. & 2015 & Holanda \\
\hline YouTube Premium & YouTube LLC & Alphabet Inc. & 2018 & EE.UU. \\
\hline
\end{tabular}




\begin{tabular}{|c|c|c|c|c|}
\hline Servicio & Proveedor & Propietario & \multicolumn{2}{|c|}{$\begin{array}{c}\text { Lanzamiento } \\
\text { y país } \\
\text { establecimiento }\end{array}$} \\
\hline MUBI España & \multicolumn{2}{|c|}{ MUBI Inc. (participada por Huanxi Media) } & 2010 & EE.UU. \\
\hline Sky & Sky UK Ltd. & $\begin{array}{l}\text { Fox Corp. (The Walt } \\
\text { Disney) }\end{array}$ & 2017 & $\begin{array}{l}\text { Reino } \\
\text { Unido }\end{array}$ \\
\hline EROSNOW & Eros International Ltd. & Eros International Plc. & 2012 & India \\
\hline spuul & \multicolumn{2}{|l|}{ Spuul Pte Ltd. } & 2012 & Singapur \\
\hline Pantaflix & Pantaflix Technologies & $\begin{array}{l}\text { Blackmars Capital } \\
\text { Gmbh. }\end{array}$ & 2016 & Alemania \\
\hline realeyz & \multicolumn{2}{|l|}{ EYZ Media GmbH. } & 2009 & Alemania \\
\hline iROKOtv & iROKO Ltd. & iROKO Partners & 2013 & Nigeria \\
\hline Vimeo on Demand & Vimeo LLC & IAC/Interactive Corp. & 2009 & EE.UU. \\
\hline Crunchyroll & Crunchyroll & Otter Media Holdings LLC & 2013 & EE.UU. \\
\hline WolfeOnDemand & \multicolumn{2}{|c|}{ Wolfe Vídeo (distribuidora; Kathy Wolfe fundadora) } & 2012 & EE.UU. \\
\hline IFFR Unleashed & \multicolumn{2}{|c|}{ International Film Festival Rotterdam } & 2018 & $\begin{array}{l}\text { Países } \\
\text { Bajos }\end{array}$ \\
\hline FilmDoo & \multicolumn{2}{|c|}{ FilmDoo Ltd (Sucharitkul y Page fundadores) } & 2014 & $\begin{array}{l}\text { Reino } \\
\text { Unido }\end{array}$ \\
\hline Spamflix & \multicolumn{2}{|l|}{ Spamflix Lda. } & 2018 & Portugal \\
\hline Full Moon & \multicolumn{2}{|l|}{ Full Moon Features Ltd. } & 2013 & EE.UU. \\
\hline TROMA NOW & \multicolumn{2}{|l|}{ Troma Entertainment Inc. } & 2016 & EE.UU. \\
\hline
\end{tabular}

Nota: allí donde proveedor y propietario comparten denominación, ambas columnas se han fusionado.

Fuente: elaboración propia a partir de MAVISE (Observatorio Audiovisual Europeo), el sitio web La Pantalla Digital, e información de las plataformas.

Para algunos la llegada de Netflix al mercado europeo supuso un punto de inflexión en lo que a la aparición de nuevas plataformas de comercialización audiovisual bajo demanda respecta. Entre aquellas creadas a mediados de la década de 2010 por empresas establecidas en España cabe mencionar a GuiDoc, márgenes y, un poco más tarde, NYX Channel (Vodity, lanzada en abril de 2017 como una plataforma de documentales y ficción independiente, no se encuentra operativa dos años después aunque su sitio web promete un relanzamiento próximo); mientras que las gestionadas por compañías establecidas en otro país son, además de la mencionada Netflix, HBO, Sky, TROMA NOW y Pantaflix.

Más lanzamientos se han registrado en 2018 y 2019. La multiplicación de la oferta no sólo no se ha detenido, puesto que el mercado español ha asistido al desembarco de expansiones globales como la de Prime Video, Filmstruck y YouTube Premium $^{4}$, sino que profundiza su grado de especialización. Si Spamflix e IFFR

4 Habrá que ver la evolución de este servicio, ya que YouTube ha avanzado un cambio de estrategia para 2020 según el cual se abandonaría el modelo de suscripción para sus contenidos originales. Ver https://www.audiovisual451.com/ youtube-abandona-el-modelo-de-la-suscripcion-para-sus-contenidos-originales/?utm_source=Audiovisual451+New sletter\&utm_campaign=e44757de25-FECE+Momonsters\&utm_medium =email \&utm_term=0_0dbcb7eaf5e44757de25-82429881 
Unleashed nos llegan desde Europa, el mercado español ha generado recientemente, con objetivos disímiles eso sí, a FlixOlé, Mujeres de Cine y Cine Autor.

Como era de esperar, aparecen ya indicios de saturación. Y si bien es cierto que el escenario es complejo para hacer prognosis, puesto que los lanzamientos y cierres deben leerse tanto en clave local como global, el caso de Filmstruck puede ser revelador de la más que probable tendencia a la concentración que vivirá este segmento de la industria audiovisual. Creada en noviembre de 2016 en Estados Unidos, esta plataforma dependía de Turner Classic Movies (filial de Warner Media, propiedad a su vez de AT\&T) y se especializaba en cine clásico e indie. El servicio se cerró en noviembre de 2018 tal y como ocurrió con otras ofertas del grupo, también de nicho, como DramaFever, dedicada a la ficción coreana. En España sólo estuvo disponible unos meses, desde julio del mismo año. La decisión de Warner habría sido la de concentrar sus esfuerzos en la plataforma $\mathrm{HBO}$, dejando caer aquellas unidades de negocio con un margen menor o más incierto (Spangler, 2018).

\section{2. ¿Atomización empresarial vs. hegemonía global?}

A la hora de retratar y clasificar el perfil empresarial de todas estas plataformas, sintetizado de alguna manera en las tablas 4 y 5 , cabe hacer una primera distinción entre las originadas y establecidas en España y aquellas disponibles en nuestro mercado pero cuyo proveedor opera legalmente desde otro país.

Dejando de lado el caso de Rakuten TV, entre las plataformas establecidas en España cabe distinguir a su vez tres grandes tipos. En primer lugar se encuentran aquellas derivadas del negocio televisivo de grandes grupos de comunicación como Mediaset (Mitele) y Atresmedia (Atresplayer). Presentan una clara orientación a la explotación ampliada de títulos previamente adquiridos y/o producidos para su negocio central, la televisión en abierto, por lo que su oferta generalista se encuentra accesible de modo gratuito, con los contenidos especializados mediados por el pago.

Un segundo grupo es el de aquellas plataformas surgidas del interés y/o la colaboración entre productoras y distribuidoras nacionales: Filmin y Cineclick, para el caso de las empresas más consolidadas; Feelmakers, Benecé, márgenes, Cine Autor, IN-EDIT, para el caso de agentes pequeños y medianos. Destacan mayoritariamente por su especialización en la oferta, con estrategias de posicionamiento claras por segmentos de público, con las plataformas más pequeñas organizadas alrededor del alquiler de sus títulos - en combinación con contenidos gratuitos, que se usan como reclamo, financiados cuando es posible por publicidad - y las demás centradas en modelos mixtos que combinan la suscripción con el alquiler.

En tercer lugar pueden detectarse emprendimientos comerciales de escala pequeña (ADNstream, Televeo, GuiDoc, NYX) y mediana (FlixOlé, Tus Telenovelas), presumiblemente todos irrelevantes desde el punto del vista del negocio, aunque algunos lo son por su reciente creación (Mujeres de Cine). En este subgrupo conviven diferentes modelos de negocio, escorados hacia el freemium, sobre todo cuando se trata de una oferta generalista, o el alquiler de títulos. Una excepción en toda regla, pendiente de conocer su evolución, es el caso de FlixOlé, que es el único que destaca, no tanto por su oferta súper especializada, que también definiría a GuiDoc o NYX, sino por el volumen de su catálogo (básicamente todos los títulos gestionados por Enrique Cerezo) y su atractivo precio de suscripción. 
Tabla 4. Plataformas audiovisuales de pago bajo demanda disponibles en España OTT - Oferta (abril de 2019).

\begin{tabular}{|c|c|c|c|c|}
\hline \multirow[t]{2}{*}{ Servicio } & \multirow{2}{*}{$\begin{array}{c}\text { Tipo de } \\
\text { contenido }\end{array}$} & \multicolumn{2}{|c|}{$\begin{array}{l}\text { Número de } \\
\text { referencias }\end{array}$} & \multirow[t]{2}{*}{ Detalle catálogo } \\
\hline & & Cine* & Series** & \\
\hline \multicolumn{5}{|c|}{ Establecidos en España } \\
\hline $\begin{array}{l}\text { Rakuten TV } \\
\text { (ES) }\end{array}$ & Generalista & 5.217 & 35 & $\begin{array}{l}\text { Gran importancia a blockbusters y } \\
\text { clásicos premiados. }\end{array}$ \\
\hline Mitele & Generalista & 153 & 78 & $\begin{array}{l}\text { Dominado por la ficción financiada por } \\
\text { el Grupo Mediaset. Combina contenidos } \\
\text { gratuitos y de pago. }\end{array}$ \\
\hline Atresplayer & Generalista & 1.954 & 117 & $\begin{array}{l}\text { El abono incluye series y programas de } \\
\text { televisión del Grupo Atresmedia, sin } \\
\text { anuncios. Combina contenidos gratuitos } \\
\text { y de pago. }\end{array}$ \\
\hline Filmin & Cine & 5.899 & 112 & $\begin{array}{l}\text { Producciones independientes, cine de autor, } \\
\text { clásicos y documentales, y series. También } \\
\text { algunos estrenos comerciales. }\end{array}$ \\
\hline FlixOlé & Cine & 7.000 & 1.500 & $\begin{array}{l}\text { Especialización en cine español. Clásicos } \\
\text { remasterizados. Sin estrenos comerciales. } \\
\text { Número de referencias aproximado. }\end{array}$ \\
\hline Cineclick & Generalista & 1.365 & - & Cine comercial, sin estrenos. \\
\hline ADNStream & Generalista & \multicolumn{2}{|c|}{160} & $\begin{array}{l}\text { Variopinta (con secciones como Infantil o } \\
\text { Yoga), sin estrenos. Combina contenidos } \\
\text { gratuitos y de pago. }\end{array}$ \\
\hline Televeo & Generalista & \multicolumn{2}{|c|}{192} & $\begin{array}{l}\text { Variopinta, sin estrenos. Combina } \\
\text { contenidos gratuitos y de pago. }\end{array}$ \\
\hline Benecé online & Cine & \multicolumn{2}{|c|}{52} & $\begin{array}{l}\text { Contenidos cuyo denominador común es } \\
\text { haber sido producidos por el proveedor } \\
\text { del servicio. }\end{array}$ \\
\hline Feelmakers & Documental & \multicolumn{2}{|c|}{368} & $\begin{array}{l}\text { Documentales, animación y } \\
\text { cortometrajes. }\end{array}$ \\
\hline $\begin{array}{l}\text { Tus Telenovelas } \\
\text { Online }\end{array}$ & Ficción TV & - & 1.000 & $\begin{array}{l}\text { Especializada en telenovelas. Combina } \\
\text { contenidos gratuitos y de pago. Número } \\
\text { de referencias aproximado. }\end{array}$ \\
\hline Mujeres de Cine & Cine & 48 & - & $\begin{array}{l}\text { Cine español realizado por mujeres. } \\
\text { Estrenos. En configuración dado su } \\
\text { lanzamiento en marzo de } 2019 .\end{array}$ \\
\hline IN-EDIT TV & Documental & 176 & - & $\begin{array}{l}\text { Documental musical. Vinculado al } \\
\text { festival In-Edit. }\end{array}$ \\
\hline márgenes & Cine & 85 & - & $\begin{array}{l}\text { Cine español y latinoamericano } \\
\text { alternativo. }\end{array}$ \\
\hline NYX Channel & Cine & 29 & - & Cine clásico exclusivamente, subtitulado. \\
\hline
\end{tabular}




\begin{tabular}{|c|c|c|c|c|}
\hline \multirow[t]{2}{*}{ Servicio } & \multirow{2}{*}{$\begin{array}{l}\text { Tipo de } \\
\text { contenido }\end{array}$} & \multicolumn{2}{|c|}{$\begin{array}{l}\text { Número de } \\
\text { referencias }\end{array}$} & \multirow[t]{2}{*}{ Detalle catálogo } \\
\hline & & Cine* & Series** & \\
\hline $\begin{array}{l}\text { Microsoft Store } \\
\text { - Movies \& TV } \\
\text { (ES) }\end{array}$ & Generalista & 3.511 & 140 & \multirow[t]{2}{*}{$\begin{array}{l}\text { Producciones estadounidenses } \\
\text { fundamentalmente; blockbusters. }\end{array}$} \\
\hline $\begin{array}{l}\text { Google Play } \\
\text { Movies (ES) }\end{array}$ & Generalista & 9.707 & 117 & \\
\hline $\begin{array}{l}\text { iTunes Store } \\
\text { (ES) }\end{array}$ & Generalista & 8.527 & 117 & \multirow{2}{*}{$\begin{array}{l}\text { Producciones estadounidenses } \\
\text { fundamentalmente; blockbusters. } \\
\text { Algunos contenidos gratuitos. }\end{array}$} \\
\hline $\begin{array}{l}\text { Play Station } \\
\text { Store }\end{array}$ & Generalista & 3.102 & 31 & \\
\hline $\begin{array}{l}\text { Prime Video } \\
\text { (ES) }\end{array}$ & Generalista & 1.186 & 257 & $\begin{array}{l}\text { Algunos estrenos comerciales. Producciones } \\
\text { propias. Acceso a través de la suscripción } \\
\text { anual a los servicios de Amazon Premium. }\end{array}$ \\
\hline $\begin{array}{l}\text { YouTube } \\
\text { Movies }\end{array}$ & Generalista & \multicolumn{2}{|c|}{$\mathrm{s} / \mathrm{d}$} & $\begin{array}{l}\text { Producciones estadounidenses } \\
\text { fundamentalmente; algunos blockbusters. }\end{array}$ \\
\hline HBO España & Generalista & 623 & 267 & $\begin{array}{l}\text { Producciones propias y estadounidenses } \\
\text { fundamentalmente. }\end{array}$ \\
\hline Netflix España & Generalista & 2.238 & 937 & $\begin{array}{l}\text { Producciones estadounidenses } \\
\text { fundamentalmente. Algunos blockbusters. } \\
\text { Producciones propias (sello Netflix } \\
\text { Originales). }\end{array}$ \\
\hline MUBI España & Cine & 30 & - & $\begin{array}{l}\text { Selección de } 30 \text { obras disponibles cada } \\
\text { mes. Cine de autor e internacional. } \\
\text { Adicionalmente, selección curada de } \\
\text { obras para alquiler. }\end{array}$ \\
\hline Sky & Generalista & 288 & 101 & $\begin{array}{l}\text { Producciones estadounidenses } \\
\text { fundamentalmente; algunos blockbusters. } \\
\text { Producciones propias (sello Sky Original). } \\
\text { El abono incluye además acceso a canales } \\
\text { de televisión en línea y a acontecimientos } \\
\text { deportivos. }\end{array}$ \\
\hline EROSNOW & Cine & \multicolumn{2}{|c|}{12.000} & $\begin{array}{l}\text { Cine indio, especialmente de Bollywood. } \\
\text { Multilingüe. Producciones propias (sello } \\
\text { Eros Originals). La suscripción incluye } \\
\text { contenidos musicales (no contabilizados } \\
\text { aquí). Número de referencias aproximado. }\end{array}$ \\
\hline Pantaflix & Generalista & \multicolumn{2}{|c|}{23.000} & $\begin{array}{l}\text { Producciones europeas y } \\
\text { estadounidenses, muchas vinculadas a } \\
\text { la productora Pantaleon (también del } \\
\text { proveedor del servicio). Multilingüe. } \\
\text { Número de referencias aproximado. }\end{array}$ \\
\hline realeyz & Cine & 2.300 & - & $\begin{array}{l}\text { Cine internacional independiente. } \\
\text { Multilingüe. Número de referencias } \\
\text { aproximado. }\end{array}$ \\
\hline
\end{tabular}




\begin{tabular}{|c|c|c|c|c|}
\hline \multirow{2}{*}{ Servicio } & \multirow{2}{*}{$\begin{array}{c}\text { Tipo de } \\
\text { contenido }\end{array}$} & \multicolumn{2}{|c|}{$\begin{array}{l}\text { Número de } \\
\text { referencias }\end{array}$} & \multirow{2}{*}{ Detalle catálogo } \\
\hline & & Cine* & Series** & \\
\hline $\begin{array}{l}\text { Vimeo on } \\
\text { Demand }\end{array}$ & Cine & \multicolumn{2}{|c|}{2.000} & $\begin{array}{l}\text { Producciones independientes de formatos } \\
\text { variados y gran calidad; además de propias } \\
\text { (sello Vimeo Originals). Estimación de } \\
\text { catálogo global, s/d para España. }\end{array}$ \\
\hline WolfOnDemand & Otros & 106 & - & $\begin{array}{l}\text { Se define como plataforma LGTB global. } \\
\text { Muchas referencias vinculadas a la } \\
\text { distribuidora Wolfe Video. }\end{array}$ \\
\hline IFFR Unleashed & Cine & 277 & - & $\begin{array}{l}\text { Títulos seleccionados a partir del Festival } \\
\text { Internacional de Cine de Róterdam (IFFR). }\end{array}$ \\
\hline FilmDoo & Cine & 384 & - & $\begin{array}{l}\text { Películas y cortos de cine internacional } \\
\text { independiente. Multilingüe. }\end{array}$ \\
\hline Spamflix & Otros & 34 & - & $\begin{array}{l}\text { Se definen como especializado en cine } \\
\text { 'difícil de clasificar': geeky, transgresor, } \\
\text { nonsense... catálogo en configuración } \\
\text { debido a su lanzamiento en noviembre de } \\
\text { 2018. Incluye acceso a videojuegos. }\end{array}$ \\
\hline Full Moon & Otros & 370 & - & Cine de ciencia ficción y horror. Serie B. \\
\hline TROMA NOW & Otros & $\mathrm{s} / \mathrm{d}$ & - & Cine de horror 'gore'. Serie B. \\
\hline
\end{tabular}

Notas: (*) incluye cortos, documentales y TV movies; (**) incluye miniseries.

Fuente: elaboración propia a partir de MAVISE (Observatorio Audiovisual Europeo), los sitios web JustWatch y La Pantalla Digital, e información de las plataformas.

Tabla 5. Plataformas audiovisuales de pago bajo demanda disponibles en España OTT - Modelo de negocio (abril de 2019).

\begin{tabular}{|c|c|c|c|c|c|}
\hline \multirow{2}{*}{ Servicio } & \multicolumn{3}{|c|}{ Comercialización (desde/ €)* } & \multirow{2}{*}{$\begin{array}{c}\text { Modelo } \\
\text { de negocio }\end{array}$} & \multirow{2}{*}{ Sitio web } \\
\hline & Alquiler & Suscripción & Venta & & \\
\hline \multicolumn{6}{|c|}{ Establecidos en España } \\
\hline $\begin{array}{l}\text { Rakuten TV } \\
\text { (ES) }\end{array}$ & 0.99 & 6.99 & 7.99 & TVoD, SVoD & rakuten.tv \\
\hline Mitele & 1.45 & - & - & Freemium & mitele.es \\
\hline Atresplayer & 0.99 & 2.99 & - & Freemium & atresplayer.com \\
\hline Filmin & 1.95 & 7.99 & - & SVoD, PVoD & filmin.es \\
\hline FlixOlé & - & 2.99 & - & SVoD & flixole.com \\
\hline Cineclick & 0.99 & 9.95 & - & $\begin{array}{l}\text { SVoD, TVoD, } \\
\text { AVoD }\end{array}$ & cineclick.com \\
\hline Televeo & 1.00 & - & - & Freemium & televeo.com \\
\hline Benecé online & 0.50 & - & - & TVoD, SVoD & benece.es/online \\
\hline
\end{tabular}




\begin{tabular}{|c|c|c|c|c|c|}
\hline \multirow{2}{*}{ Servicio } & \multicolumn{3}{|c|}{ Comercialización (desde/ $€$ )* } & \multirow{2}{*}{$\begin{array}{c}\text { Modelo } \\
\text { de negocio }\end{array}$} & \multirow{2}{*}{ Sitio web } \\
\hline & Alquiler & Suscripción & Venta & & \\
\hline GuiDoc & - & 5.00 & - & SVoD & guidedoc.tv \\
\hline $\begin{array}{l}\text { Tus Telenovelas } \\
\text { Online }\end{array}$ & - & 5.99 & - & Freemium & $\begin{array}{l}\text { tustelenovelasonline. } \\
\text { com }\end{array}$ \\
\hline Mujeres de Cine & 0.95 & - & - & TVoD, AVoD & $\begin{array}{l}\text { vod.mujeresdecine. } \\
\text { com }\end{array}$ \\
\hline Cine Autor & 2.50 & - & - & TVoD, AVoD & cineautor.es \\
\hline IN-EDIT TV & 1.50 & - & - & TVoD & in-edit.tv \\
\hline márgenes & 2.20 & - & - & TVoD, AVoD & márgenes.org \\
\hline NYX Channel & 1.49 & - & - & SVoD & nyxchannel.com \\
\hline \multicolumn{6}{|c|}{ Establecidos fuera de España } \\
\hline $\begin{array}{l}\text { Microsoft Store } \\
\text { - Movies \& TV } \\
\text { (ES) }\end{array}$ & 2.45 & - & 9.99 & TVoD & $\begin{array}{l}\text { microsoft.com/es-es/ } \\
\text { store/movies-and-tv }\end{array}$ \\
\hline $\begin{array}{l}\text { Google Play } \\
\text { Movies (ES) }\end{array}$ & 0.99 & - & 7.99 & TVoD & $\begin{array}{l}\text { play.google.com/store/ } \\
\text { movies?hl=en }\end{array}$ \\
\hline $\begin{array}{l}\text { iTunes Store } \\
\text { (ES) }\end{array}$ & 2.99 & - & 9.99 & TVoD, AVoD & $\begin{array}{l}\text { itunes.apple. } \\
\text { com/es/genre/ } \\
\text { pel\%C3\%ADculas/ } \\
\text { id33 }\end{array}$ \\
\hline $\begin{array}{l}\text { Play Station } \\
\text { Store }\end{array}$ & 0.99 & - & 0.99 & TVoD, AVoD & $\begin{array}{l}\text { store.playstation. } \\
\text { com/es-es/grid/ } \\
\text { STORE-MSF75508- } \\
\text { MOVALLMOVIES/1 }\end{array}$ \\
\hline $\begin{array}{l}\text { Prime Video } \\
\text { (ES) }\end{array}$ & - & $12.99 /$ año & - & SVoD & primevideo.com \\
\hline $\begin{array}{l}\text { YouTube } \\
\text { Movies }\end{array}$ & 2.99 & - & 2.99 & TVoD & youtube.com/movies \\
\hline HBO España & - & 7.99 & - & SVoD & hboespana.com \\
\hline Netflix España & - & 7.99 & - & SVoD & netflix.com \\
\hline $\begin{array}{l}\text { YouTube } \\
\text { Premium }\end{array}$ & - & 11.99 & - & Freemium & $\begin{array}{l}\text { www.youtube.com/ } \\
\text { premium }\end{array}$ \\
\hline Sky & - & 6.99 & - & SVoD & sky.es \\
\hline EROSNOW & - & 5.99 & . & SVoD & erosnow.com \\
\hline Spuul & - & 4.99 & - & SVoD, AVoD & spuul.com \\
\hline Pantaflix & 0.99 & - & - & TVoD & pantaflix.com \\
\hline realeyz & - & 5.50 & - & SVoD & realeyz.de/en \\
\hline iROKOtv & - & $\begin{array}{l}9.99(3 \\
\text { meses })\end{array}$ & - & SVoD & irokotv.com \\
\hline $\begin{array}{l}\text { Vimeo on } \\
\text { Demand }\end{array}$ & 0.99 & - & 2.47 & TVoD, AVoD & vimeo.com/ondemand \\
\hline
\end{tabular}




\begin{tabular}{|l|c|c|c|l|l|}
\hline \multirow{2}{*}{ Servicio } & \multicolumn{2}{|c|}{ Comercialización (desde/ $€$ )* } & \multicolumn{1}{c|}{$\begin{array}{c}\text { Modelo } \\
\text { de negocio }\end{array}$} & \multicolumn{1}{|c|}{ Sitio web } \\
\cline { 2 - 5 } & Alquiler & Suscripción & Venta & \multicolumn{1}{|c|}{} \\
\hline WolfOnDemand & 3.99 & - & 4.99 & TVoD & wolfeondemand.com \\
\hline IFFR Unleashed & 1.00 & - & - & TVoD & iffrunleashed.com \\
\hline FilmDoo & 1.49 & - & - & TVoD, AVoD & filmdoo.com \\
\hline Spamflix & 4.00 & - & - & TVoD & spamflix.com \\
\hline Full Moon & - & $6.20^{* *}$ & - & SVoD & $\begin{array}{l}\text { fullmoonstreaming. } \\
\text { com }\end{array}$ \\
\hline TROMA NOW & - & $5.30^{* *}$ & - & SVoD & watch.troma.com \\
\hline
\end{tabular}

Notas: (*) salvo que se indique otra cosa, el alquiler y la venta refieren al precio en $€$ por unidad, mientras que la suscripción indica el abono mensual; $(* *)$ la contratación se efectúa en dólares estadounidenses.

Fuente: elaboración propia a partir de MAVISE (Observatorio Audiovisual Europeo), los sitios web JustWatch y La Pantalla Digital, e información de las plataformas.

Dentro del grupo de iniciativas establecidas fuera de España, la oferta estaba conformada, a abril de 2019, por un conjunto de plataformas entre las cuales las más importantes tienen su origen en Estados Unidos y desembarcaron en España a partir de finales de 2015, en el contexto de su estrategia de expansión europea. En este conjunto, como en el anterior, cabe distinguir distintas iniciativas que pueden organizarse fundamentalmente en tres tipos.

En primer lugar hemos de mencionar a las de alcance global, vinculadas a los denominados GAFAM (Google Play, iTunes Vídeo, Amazon Instant Video, YouTube Movies/Premium y Microsoft Movies \& TV), o a corporaciones mundiales de medios y entretenimiento (Sky, HBO, Play Station Store). El caso singular en este subgrupo es el de Netflix, presente en prácticamente todo el mundo pero sin las ramificaciones empresariales de las demás. Estas plataformas comercializan catálogos generalistas a través de modelos de negocio muy bien definidos: o bien el de la tienda online (alquiler y venta), o bien el de la suscripción.

En un segundo nivel pueden ubicarse un buen número de plataformas que podríamos calificar como multinacionales (aunque muchas manifiesten aspiraciones globales), en general vinculadas a grupos multimedia, cuya presencia geográfica más allá de su país de establecimiento depende de acuerdos estratégicos de distribución. En este subgrupo pueden incluirse Vimeo, Crunchyroll, MUBI, EROSNOW, spuul, realeyz, Pantaflix e iROKOtv. Aunque la firma esté legalmente establecida en España - porque su origen está en una iniciativa que surgió en Barcelona como Waki TV consideramos también parte de este subgrupo a Rakuten TV, propiedad del llamado "Amazon japonés".

Se trata, por un lado, de plataformas con catálogos generalistas, en buena medida comercializados bajo modelos de negocio mixtos, y, por otro, de plataformas de SVoD centradas en aproximaciones especializadas al cine, sea este más o menos comercial (Bollywood, Nollywood, manga...). Aunque bien es cierto que los casos de MUBI y Vimeo on Demand son del todo singulares. El primero por presentar un modelo de negocio único en su tipo, el segundo por ser parte de una compañía que a su vez 'aloja' plataformas (caso de WolfeOnDemand). 
Finalmente, cabe la siguiente distinción al interior de un tercer tipo de plataformas cuyo común denominador es tener un catálogo de referencias limitado pero súper especializado. Aquí encontramos, por un lado, a empresas de producción y/o distribución, especializadas en algún tipo de cine, que se decantan por la opción del alquiler/ venta o por la suscripción (WolfOnDemand, Full Moon y TROMA); y, por otro, lado encontramos iniciativas de menor talla, orientadas también a nichos (IFFR Unleashed, FilmDoo), con un futuro incierto para las de más reciente creación (Spamflix).

En definitiva, podría concluirse que las 'plataformas españolas' evidencian una clara atomización empresarial y que entre las mismas sólo parecen ser viables las vinculadas a grupos de comunicación y empresas solventes de la industria cinematográfica. Filmin, en definitiva, nace de la colaboración de productoras, distribuidoras y exhibidoras españolas. No existen indicios de que todas las demás sean económicamente relevantes y, por tanto, está por ver si sobreviven. Por su parte, aunque las plataformas establecidas fuera de España no son pocas, los datos disponibles señalan la hegemonía, de hecho global, de aquellas aquí clasificadas como vinculadas a los GAFAM o a corporaciones mundiales de medios y entretenimiento. Además de Netflix, claro está.

Todos los datos sugieren, de hecho, la prevalencia de esta última plataforma entre los consumidores españoles de audiovisual de pago bajo demanda ofrecido OTT. Más allá del número de abonados (tabla 6), otros indicadores avalan esta afirmación. Según Parrot Analytics (2019), si se consideran todos los títulos originales estrenados en línea en España en 2018, los de Netflix acaparan casi el 75\% del total de la demanda. Asimismo, un estudio reciente publicado por IAB Spain (2018) afirma que, después de YouTube, Netflix es la plataforma de contenido de vídeo en línea más conocida, tanto de forma espontánea como sugerida, entre los más de 24 millones de consumidores de estos contenidos, que tienen entre 16 y 65 años.

Tabla 6. Principales plataformas audiovisuales de pago en España (hogares abonados, junio 2018).

\begin{tabular}{|l|r|}
\hline Movistar+ en dispositivos & 2.200 .000 \\
\hline Netflix & 2.000 .000 \\
\hline Vodafone TV online & 950.000 \\
\hline App Orange TV & 741.000 \\
\hline Amazon Prime Video & 648.000 \\
\hline HBO & 476.000 \\
\hline beIN CONNECT & 375.000 \\
\hline Rakuten TV & 153.000 \\
\hline Sky & 115.000 \\
\hline Otras & 98.000 \\
\hline
\end{tabular}

Fuente: CNMC, Panel de Hogares (posible respuesta múltiple). 


\section{3. ¿Hay un 'efecto Netflix'?}

Cabe la pregunta de cuál es la importancia real, además de potencial, que tienen las plataformas aquí estudiadas, dado que es muy difícil encontrar cifras de facturación e ingresos de estos emprendimientos empresariales, muchas veces camufladas en el conjunto de sus operaciones. La consulta de diversos informes sectoriales puede al menos dar pistas del orden de magnitudes en juego.

Según el Informe Anual del Sector TIC y de los Contenidos en España 2018 (ONTSI, 2018), la cifra total de negocio de las actividades de distribución cinematográfica y de vídeo ascendió a 796 millones de euros en 2017, siendo aquellas categorías relacionadas con la distribución bajo demanda y/o en streaming las más modestas del conjunto.

Por su parte, las cifras de la International Video Federation (2018) demuestran el aumento sostenido, y pronunciado desde 2016, en el gasto dedicado a consumir vídeo OTT en España: 222,4 millones de euros en 2017, en un cómputo que incluye también la venta de películas y series. Las cifras de la CNMC (2018b) sobre la distribución de los hogares abonados dan cuenta también de la expansión del segmento.

Así, al añadir a la descripción de ofertas y modelos de negocio presentada algunas informaciones sobre las magnitudes del mercado audiovisual de pago bajo demanda, podemos afirmar que el audiovisual ofrecido OTT se está configurando como un segmento en consolidación pero controlado por agentes de actuación trasnacional, alcance global y origen estadounidense. Ya que si bien existen iniciativas nacidas en España, la presencia y poder de actores como Netflix y Amazon no deja de ir en aumento. El próximo lanzamiento de Apple TV+ en España anunciado como la plataforma para comprar o alquilar hasta 100.000 títulos, que incluirá también canales premium, así como la puesta en marcha de Disney+ en Estados Unidos (noviembre 2019), que se prevé disponible en todo el mundo en los dos años subsiguientes, no hará más que agudizar este diagnóstico.

Dicho esto, hay que señalar que el peso de estos agentes en el conjunto del mercado televisivo español es todavía relativo. Siempre considerando datos de la CNMC (2018a, 2018b), la TDT continua siendo la modalidad de televisión hegemónica, en términos de audiencia y facturación, controlada por el duopolio Mediaset y Atresmedia; y la televisión de pago está liderada por los servicios de IPTV ofrecidos por los operadores de telecomunicaciones, con Movistar a la cabeza.

Es muy probable, sin embargo, que la evolución de las plataformas aquí estudiadas acabe cuestionando tanto la supervivencia de las de menor talla como los equilibrios del sistema audiovisual en general. Y no sólo por las proyecciones optimistas de ingresos para el segmento de vídeo OTT, tanto SVOD como TVoD, que hacen algunas consultoras (de 142 millones de euros en 2018 se pasaría a 234 millones de euros en 2022; PwC, 2018a). Sino porque la competencia parece que va a darse en la generación de contenidos propios como factor diferenciador, especialmente en series (Fundación Telefónica, 2017), dado que existe evidencia de que esto atrae más clientes (Prince y Greenstein, 2018).

Estaríamos asistiendo pues a un proceso en el que no sólo se multiplican y ganan terreno agentes que hace poco más de diez años no existían - plataformas de audiovisual bajo demanda que operan OTT - sino que aquellos que entre los mismos se configuran como hegemónicos se encuentran establecidos fuera de España, actúan globalmente, y han iniciado un movimiento productivo que va de la mera distribu- 
ción a la producción (si es que no presentan dicha integración ya). Este no es únicamente el caso de Netflix, con sus abultadas cifras de inversión (según Goldman Sachs sus inversiones superarán los 22.500 millones de dólares en todo el mundo para 2022). Amazon también trabaja en la misma dirección.

En España, este movimiento se refleja en datos significativos como los siguientes (PwC, 2018b; Mezzera, 2019): si, por un lado, además de la muy publicitada instalación de lo que Netflix ha presentado como su primer hub europeo de producción audiovisual en Madrid, ha trascendido que Amazon y Viacom International Studios irán por el mismo camino, por otro lado, se confirma la multiplicación de la producción de ficción de los últimos años (de 38 series originales que se estrenaron en 2015 hemos pasado a 50 en 2018 ), que está previsto que alcance los 78 títulos cuando acabe el 2019 (57 estrenos y 21 nuevas temporadas). Entre Netflix, HBO y Amazon se dará cobijo a 22 de estos títulos.

\section{A modo de conclusión}

Evidentemente es difícil concluir de modo tajante sobre las oportunidades y amenazas que las plataformas audiovisuales de pago bajo demanda OTT pueden suponer para la diversidad de/en la industria audiovisual española. Un balance necesariamente incompleto, por falta de algunos datos relevantes, podría indicar, en primer lugar, que el número de plataformas disponibles impide hablar de concentración empresarial. Los sucesivos lanzamientos y cierres de la última década, tanto a escala local como internacional, apuntan en cambio en esa dirección. En segundo lugar, frente a las miles de referencias que incorporan algunos catálogos, sigue pendiente de comprobar que los mismos presentan suficientes diferencias en términos de variedad, balance y disparidad. Aunque son pocas, las evidencias disponibles señalan por el contrario el predominio de los contenidos de origen estadounidense (ver por ejemplo Fontaine y Grece, 2016). Finalmente, frente al argumento de que el consumidor puede acceder a y elegir entre, como nunca antes, un elevado y variado número de plataformas, hay que recordar que dicho acceso está mediado por el pago.

Y si bien es cierto, más allá de las visiones triunfalistas, que existen oportunidades reales para que se consolide el crecimiento que está experimentado el mercado español de producción audiovisual (especialmente en ficción), no menos verdadero es que los equilibrios del conjunto del sistema se verán afectados, a corto y medio plazo.

Por ejemplo, la competencia feroz ya establecida entre las plataformas aquí estudiadas y, a su vez, con el resto de ofertas de pago, está conduciendo a cambios en la tarificación de los servicios que comienzan a desglosarse en multitud de 'planes' (básico, estándar, premium....), así como a la reconfiguración permanente de las ofertas (número y tipo de títulos) cuyo dinamismo repercute en el grado de producción original propia y/o española ofrecido en formas no siempre fáciles de rastrear.

En un plano más general, asimismo, aumenta la (ya existente) fragmentación de audiencias, que se reparten entre más proveedores (muchos de los cuales, sin embargo, no comparten sus datos); empieza a detectarse el fenómeno conocido en Estados Unidos como cord cutting, que refiere a la cancelación de suscripciones a las ofertas de televisión de pago de operadores pre-existentes (cable, satélite e incluso IPTV); y 
se recrudece la puja por la adquisición de derechos de emisión de los contenidos internacionalmente más populares (series especialmente ${ }^{5}$ ).

Por último, pero no en último lugar, cabe señalar que asistimos a la consolidación de una sociedad cada vez más desequilibrada y segmentada en sus consumos audiovisuales (Bustamante, 2018): televisión para los más desfavorecidos en los modelos abiertos tradicionales (TDT), servicios audiovisuales 'gratuitos' con publicidad para los jóvenes pobres más activos (YouTube), paquetes de canales de pago cada vez más abundantes y sofisticados para los pudientes (Movistar+), video on demand en todo tipo de redes para los ricos y activos (Netflix, HBO...); en definitiva, dosis desiguales de contenidos diferenciados que redoblarán las desigualdades sociales.

\section{Referencias bibliográficas}

Albornoz, L. A. y García Leiva, M. T. (2017a): Diversidad e industria audiovisual: el desafio cultural del siglo XXI, México D.F., Fondo de Cultura Económica (FCE).

Albornoz, L. A. y García Leiva, M. T. (2017b): El audiovisual en la era digital. Políticas y estrategias para la diversidad, Madrid, Cátedra.

Albornoz, L. A. y García Leiva, M. T. (2019): Audiovisual Industries and Diversity. Economics and Policies in the Digital Era, Londres, Routledge.

BCG Analysis (2016): The future of Television. The impact of OTT on video production around the World, Nueva York, The Boston Consuting Group.

Bustamante, E. (2018): “Televisión en España: una década perdida”, en Díaz Nosty, B. (coord.), Diez años que cambiaron los medios: 2007-2017, Madrid, Fundación Telefónica \& Ariel, pp. 159-166.

Cabrera y Valais (2016): VOD, platforms and OTT: which promotion obligations for European Works?, IRIS Plus 2016-03, Estrasburgo, European Audiovisual Observatory.

CE (Comisión Europea) (2016): Comunicación de la Comisión al Parlamento Europeo, al Consejo, al Comité Económico y Social Europeo y al Comité de las Regiones, Las plataformas en línea y el mercado único digital. Retos y oportunidades para Europa, SWD(2016) 172 final, Bruselas, COM(2016) 288 final, 25 de mayo.

CNMC (2015): Caracterización del uso de algunos servicios over the top en España (comunicaciones electrónicas y servicios audiovisuales), Documento de Trabajo $\mathrm{n}^{\circ} 4$, diciembre, Madrid, Comisión Nacional de los Mercados y la Competencia (CNMC).

CNMC (2017): Panel de hogares del segundo trimestre de 2017. Disponible en: http://data. cnmc.es/datagraph/

CNMC (2018a): Informe Económico Sectorial de las Telecomunicaciones y el Audiovisual 2018, Madrid, Comisión Nacional de los Mercados y la Competencia (CNMC).

CNMC (2018b): Panel de hogares del segundo trimestre de 2018. Disponible en: http://data. cnmc.es/datagraph/

De Voldere, I. (Coord.) (2017): Mapping the Creative Value Chains. A study on the economy of culture in the digital age, Informe de IDEA, KEA y SMIT para la Dirección General de Educación y Cultura de la Comisión Europea, Bruselas, Comisión Europea.

5 En España, por ejemplo, la serie The Big Bang Theory, de la cadena estadounidense CBS, se puede ver completa en $\mathrm{HBO}$ porque en su día esta plataforma ganó a Netflix en la puja por sus derechos de emisión. 
DIGITAL TV RESEARCH (2018): Global SVOD subscriptions to reach 777 million. Disponible en: https://www.digitaltvresearch.com/ugc/Global\%20SVOD $\% 20$ Forecasts $\% 20$ 2018\%20TOC_sample_215.pdf

FINDANYGAME (2017): Plataformas Digitales de Cine y Series en España. Disponible en: http://www.findanygame.com/Findanygame2017.pdf

Fontaine, g. y Grece, C. (2016): Origin of films and TV content in VOD catalogues in the EU \& Visibility of films on VOD services, Estrasburgo, Observatorio Audiovisual Europeo.

Frau-Meigs, D. y Kiyindou, A. (Dirs.) (2014): Diversité culturelle à l'ère du numérique : Glossaire critique, París, Editions de La documentation française.

FUNDACIÓN TELEFÓNICA (2017): Sociedad digital en España 2017, Madrid, Fundación Telefónica \& Ariel.

García Leiva, M. T. (2016): “CCómo mapear la diversidad de la industria audiovisual en la era digital? Notas metodológicas", CIC. Cuadernos de Información y Comunicación, $\mathrm{n}^{\circ}$ 21, pp. 13-25. DOI: $10.5209 /$ CIYC.52880

García Leiva, M. T. (2017): "Desafíos y oportunidades para la diversidad del audiovisual en Internet", Política \& Sociedade: Dossiê Diversidade Cultural e Comunicação. V. 16, $\mathrm{n}^{\circ}$ 35, pp. 132-158. DOI: 10.5007/2175-7984.2017v16n35p132

Gillespie, T. (2010): “The politics of «platforms»”, New Media \& Society, 12(3), pp. 347-364.

Grece, C., Lange, A., Schneeberger, A. y Valais, S. (2015): The development of the European market for on-demand audiovisual services, Estrasburgo, European Audiovisual Observatory.

IAB SPAIN \& ELOGIA (2018): Estudio Anual Video Online 2018, Madrid, IAB Spain. Disponible en: https://iabspain.es/estudio/estudio-anual-de-vídeo-online-2018-version-completa/

IDATE (2016): Digiworld Yearbook 2016, Montpellier, IDATE.

IVF (International Video Federation) (2018): Video market: Spain. Disponible en: http:// www.ivf-vídeo.org/new/public/2018_IVF_Industry_report_Spain.pdf

Martens, B. (2016): An Economic Policy Perspective on Online Platforms, Institute for Prospective Technological Studies Digital Economy, Working Paper 2016/05, JRC101501.

Mezzera, P. (2019): "El boom de la ficción española: todo lo que se viene", TTV, 30 de abril. Disponible en: http:/www.todotvnews.com/news/El-boom-de-la-ficcin-espaola-todo-loque-se-viene.html

ONTSI (2018): Informe Anual del Sector TIC y de los Contenidos en España 2018, Madrid, Red.es. Disponible en: https:/www.ontsi.red.es/ontsi/sites/ontsi/files/InformeAnualSectorTICC2018_0.pdf

PARROT ANALYTTICS (2019): The Global Television Demand Report 2018. Disponible en: https://www.parrotanalytics.com/insights/

Prado, E. (2017): "El audiovisual online over the top: el futuro del audiovisual europeo y español", en Bustamante, E. (Coord.), Informe sobre el estado de la cultura en España 2017. Igualdad y diversidad en la era digital, Madrid, Fundación Alternativas.

Prince, J. y Greenstein, S. (2018): "Does Original Content Help Streaming Services Attract More Subscribers?", Harvard Business Review, 24 de abril. Disponible en: https://hbr. org/2018/04/does-original-content-help-streaming-services-attract-more-subscribers

PWC (2018a): Entertainment and Media Outlook 2018-2022 España, Madrid, PricewaterhouseCoopers (PwC).

PWC (2018b): La oportunidad de los contenidos de ficción en España, Madrid, PricewaterhouseCoopers (PwC).

SPANGLER (2018): “Warner Media's FilmStruck Subscription-Streaming Service to Shut Down", Variety, 26 de octubre. Disponible en: https:/variety.com/2018/digital/news/ filmstruck-shutdown-warnermedia-turner-1202998364/ 